\title{
Crossover between Equilibrium and Shear-controlled Dynamics in Sheared Liquids
}

\author{
L. Angelani ${ }^{1,2}$, G. Ruocco ${ }^{1}$, F. Sciortino ${ }^{1,2}$, P. Tartaglia ${ }^{1,2}$, and F. Zamponi ${ }^{1}$ \\ ${ }^{1}$ Dipartimento di Fisica and INFM, Università di Roma La Sapienza, P. A. Moro 2, 00185 \\ Roma, Italy \\ ${ }^{2}$ INFM - Center for Statistical Mechanics and Complexity, Università di Roma La Sapienza, $P$. \\ A. Moro 2, 00185 Roma, Italy
}

(October 30, 2018)

\begin{abstract}
We present a numerical simulation study of a simple monatomic LennardJones liquid under shear flow, as a function of both temperature $T$ and shear rate $\dot{\gamma}$. By investigating different observables we find that $i$ ) It exists a line, $T_{\dot{\gamma}}$, in the $(T-\dot{\gamma})$ plane that sharply marks the boarder between an "equilibrium" and a "shear-controlled" region for both the dynamic and the thermodynamic quantities; and ii) Along this line the structural relaxation time, $\tau_{\alpha}\left(T_{\dot{\gamma}}\right)$, is proportional to $\dot{\gamma}^{-1}$, i. e. to the typical time-scale introduced by the shear flow. Above $T_{\dot{\gamma}}$ the liquid dynamics is unaffected by the shear flow, while below $T_{\dot{\gamma}}$ both $T$ and $\dot{\gamma}$ control the particle motion.

PACS Numbers : 61.20.Lc, 64.70.Pf, 47.50.+d
\end{abstract}

Typeset using REVTEX 
The effects of the shear flow on the properties of simple liquids has recently been the object of extensive investigations [1 6]. Beside its intrinsic theoretical interest (the study of out-of-equilibrium stationary states), it has been hypothesized [7,8] that the shear flow acts as an aging-stopping mechanism, thus suggesting an interesting experimental method to study dynamics in supercooled liquids and glasses. Indeed, under shear flow, the system is in an out-of-equilibrium situation but reaches a stationary regime: similarly to equilibrium the correlation functions depend only on the time-difference. This takes place also at those temperatures for which the non-sheared system would be in an aging regime (i. e. the correlation functions depend explicitly on two times). In the common phrasing, the application of a shear flow has the effect to rejuvenate the glassy system and the aging phenomenon characteristic of glasses is stopped. The fact that some relevant properties of aging systems, like the existence of a generalized fluctuation-dissipation relation [7,9] and of an effective temperature, still hold in systems under shear flow [8,10], makes the study of sheared systems an important topic. This is particularly true in view of possible experiments aiming to probe the out-of-equilibrium dynamics of glassy systems: in aging the waiting-time dependence of correlation functions prevents the acquisition of the data with the desired statistics, while under shear correlation functions become time translation invariant.

In this work we use numerical simulations to study the effects of the shear flow on the properties of a simple Lennard-Jones model liquid. In particular we compare the temperature dependence of some physical quantities of the driven system (with different shear rates $\dot{\gamma})$ with those of the equilibrium system $(\dot{\gamma}=0)$. We find that $i)$ it exists a well-defined crossover temperature $T_{\dot{\gamma}}$, whose value depends on the shear rate $\dot{\gamma}$, below which the properties of the sheared system exhibit a marked difference from those of the equilibrium system. On the contrary, for $T>T_{\dot{\gamma}}$ the driven system is not influenced by the presence of a shear flow, and both the energy and the structural relaxation time coincide with their equilibrium values. ii) The same $T_{\dot{\gamma}}$ can be derived from the $T$-dependence of both potential energy and structural relaxation times, thus indicating the robustness of the "cross-over" temperature concept; iii) At $T_{\dot{\gamma}}$, the structural relaxation time $\tau_{\alpha}\left(T_{\dot{\gamma}}\right)$ is proportional to $\dot{\gamma}^{-1}$, i. e. to the 
typical time-scale introduced by the shear, the proportionality coefficient being an observable dependent quantity. The previous observations lead to a microscopic interpretation of the shear thinning effect, and suggest a quantitative experimental test on the temperature dependence of the non-linear viscosity in simple liquids.

The investigated system is made of $N=256$ particles interacting via a simple LennardJones potential, plus a small many-body term [11 introduced to prevent the crystallization unavoidably occurring in undercooled monatomic systems. The particles are confined in a cubic box, at density $\rho=1$ (hereafter all the quantities are expressed in reduced LJ unit), with periodic boundary condition adapted to the presence of a shear flow. The latter is applied to the system along the $x$ direction with a gradient velocity field along the $y$ axis. The molecular dynamics simulation is performed using SLLOD algorithm [12], with a Nose-Hoover thermostat for the thermal velocities. Different shear rates $\dot{\gamma}$ were studied in the range $\dot{\gamma}=10^{-1} \div \dot{\gamma}=10^{-3}$. A preliminary simulation performed at $\dot{\gamma}=0$ was used to determine the reference behavior of the equilibrium system. For all the shear rates considered, different physical quantities are analyzed as a function of temperature: energy, incoherent scattering functions, relaxation times.

In Fig. 1 we report the potential energy per particle $e$ as a function of temperature for selected values of the shear rate. The full line indicates the temperature dependence of $e$ at equilibrium $(\dot{\gamma}=0)$, which, as shown in previous work [11, 13, 14], can be described quite accurately by the Rosenfeld-Tarazona $T^{3 / 5}$ power-law [15]. The open symbols indicates the caloric data for three selected shear rates: $\dot{\gamma}=1 \cdot 10^{-1}, 4 \cdot 10^{-2}, 6 \cdot 10^{-3}$. At high temperature, one can observe a good agreement between $e(T, \dot{\gamma})$ and $e(T, \dot{\gamma}=0)=e_{e q .}(T)$. On lowering the temperature, the agreement breaks down at a $\dot{\gamma}$-dependent temperature $\left(T_{\dot{\gamma}}\right)$, below which the caloric curve of the sheared system deviate from the $T^{3 / 5}$ power-law. In particular, at low- $T, e(T, \dot{\gamma})$ is well described by a linear temperature dependence (indicated, as an example, by the dashed line for $\dot{\gamma}=4 \cdot 10^{-2}$ ). The deviation of the energy of the driven system from the equilibrium energy is evidenced in the inset of Fig. 1, where the energy difference $e-e_{e q}$. is reported as a function of temperature for the system with $\dot{\gamma}=4 \cdot 10^{-2}$. 
The existence of a linear behavior of $e(T, \dot{\gamma})$ at low $T$ and of an analytic expression for this quantity at high $T$ allows for a straightforward identification of a crossover temperature $T_{\dot{\gamma}}$ (indicated in the inset of Fig. 1 with dashed arrow).

In Fig. 2 we report the $\dot{\gamma}$-dependence of the parameters describing the caloric curves of the sheared systems: the crossover temperature $T_{\dot{\gamma}}$ and the potential energy value at $T=0$ $e_{0}$. As shown in Fig. 2a, $T_{\dot{\gamma}}$ is an increasing function of the shear rate. For small shear rate values it approaches a plateau whose value is very close to the estimated mode-coupling critical temperature $T_{M C T}\left(T_{M C T}=0.475\right.$ for this potential model [16, 17] $)$ indicated by the dashed line in Fig. 2a. A similar plateau is observed in the $\dot{\gamma}$ dependence of $e_{0}$ (see Fig. 2b). Here, for small shear rate values, $e_{0}$ reaches the value $e_{0}^{p l} \sim-6.90$. We note that the value $e_{0}^{p l}$ is higher than the lowest inherent structure energy value obtained in equilibrium simulation $e_{0}^{I S}=-7.0$ [18]. Similarly to the case of analytic mean field spin glass models [8], the plateau value can be interpreted as a threshold in the potential energy surface, above which the system is forced by the shear. However, as we are dealing with a non-mean field system, we expect that for values of $\dot{\gamma}$ small enough -well below the ones that we are able to study- the system cross the threshold. Similarly, also in Fig. 2a the existence of a plateau is only apparent and mirrors the (apparent) power-law divergence of the relaxation times predicted by the MCT. The evaluation of the crossover temperature for even smaller $\dot{\gamma}$, i. e. in a region not accessible to the simulation for CPU time reason, would have resulted in a $T_{\dot{\gamma}}$ smaller than $T_{M C T}$ as a consequence of the presence of activated processes in the investigated non-mean field system.

Further information on the effect of the shear flow can be obtained analyzing the temperature dependence of correlation functions in driven systems. For the different shear rates considered, we have calculated the incoherent scattering functions $F_{\mathbf{q}}(t)$ :

$$
F_{\mathbf{q}}(t)=\frac{1}{N} \sum_{j=1}^{N}\left\langle e^{i \mathbf{q} \cdot\left[\mathbf{r}_{j}(t)-\mathbf{r}_{j}(0)\right]}\right\rangle,
$$

where $\mathbf{r}_{j}(t)$ is the position of particle $j$ at time $t$. The wave vector $\mathbf{q}$ is that of the first peak of the static structure factor $S_{\mathbf{q}}\left(q_{\max }=7.1\right)$ along the "shear-free" direction $\left(\mathbf{q}=\left(0,0, q_{\max }\right)\right)$. 
In Fig. 3 we report the $F_{\mathbf{q}}$ for the equilibrium system (full lines) and, as an example, for the system with $\dot{\gamma}=4 \cdot 10^{-2}$ (dashed lines), for three different temperatures. At the higher reported temperature $(T=1.6)$, the $F_{\mathbf{q}}$ of the sheared system is undistinguished from the equilibrium one. At the intermediate temperature $(T=0.80)$ the full and dashed lines start to deviate one from the other, an effect that become more and more clear on lowering the temperature $(T=0.56)$. It is worth to note that -at the reported value of $\dot{\gamma}$ - the crossover temperature derived from Fig. 1 is $T_{\dot{\gamma}}=0.97$, i. e. intermediate between the the first two reported $F_{\mathbf{q}}$. From the inspection of Fig. 3, one can conclude that for $T$ smaller than $T_{\dot{\gamma}}$ (derived from $e$ ) there are no effects of the shear flow on the dynamics.

To put the previous observation on a quantitative ground, we analyze the temperature dependence of relaxation time $\tau_{\alpha}$, defined as the time at which $F_{\mathbf{q}}$ reach $1 / e$-th of its nonergodicity factor (the apparent plateau value). In Fig. 4 the power law fit to the equilibrium $(\dot{\gamma}=0)$ relaxation times is reported as full line, together with the relaxation times for three selected shear rates: $\dot{\gamma}_{1}=1 \cdot 10^{-1}$ (full diamonds), $\dot{\gamma}_{2}=4 \cdot 10^{-2}$ (full circles) and $\dot{\gamma}_{3}=6 \cdot 10^{-3}$ (open circles). In the same figure are also indicated by dashed lines the crossover temperatures derived from Fig. 1 for the three selected shear rates. Similarly to the thermodynamic quantities, also the dynamics follow a simple behavior: at a fixed $\dot{\gamma}$ value, for $T$ larger than a certain threshold the dynamics of the sheared system is undistinguished from the equilibrium one. Below the threshold the relaxation time flattens and no longer follows the steep increase associate to the slowing down of the dynamics which precedes the glass transition. It is important to emphasize that the crossover temperature, defined from the dynamics is found to be the same as that derived from thermodynamic data.

The existence of a well defined crossover temperature $T_{\dot{\gamma}}$, as evidenced by the above results, suggests the following scenario: for $T>T_{\dot{\gamma}}$ the sheared system is not affected by the shear flow because the structural relaxation process of the equilibrium system acts on a time-scale $\left(\tau_{\alpha}\right)$ that is faster than the one introduced by the shear flow; on lowering the temperature $\tau_{\alpha}$ increases and -for temperatures close to $T_{\dot{\gamma}}$ - becomes comparable to the time scale introduced by the shear (proportional to the inverse of shear rate $\dot{\gamma}^{-1}$ ). For $T<T_{\dot{\gamma}}$ 
the shear starts to modify both the static and the dynamic properties of the system. In the following, we refer to the high temperature regime $\left(T>T_{\dot{\gamma}}\right)$ as " $\alpha$-dominated" (equilibrium region) and the low temperature regime $\left(T<T_{\dot{\gamma}}\right)$ as "shear-dominated". Below $T_{\dot{\gamma}}$, in the "shear-dominated" region, the system, still remaining in a stationary state, "freezes" (in the sense that relevant processes governing the relaxations become those induced by the shear and then only weakly temperature dependent). Dynamic behavior are much less affected by further decreases of temperature and the relaxation time reaches a finite value for $T \rightarrow 0$. The previous scenario implies the existence of a strong relation between the equilibrium relaxation time at the crossover temperature and $\dot{\gamma}^{-1}$. In Fig. 5 we compare $\tau_{\alpha}(T)$ plotted as a function of the temperature and the inverse shear rate $\dot{\gamma}^{-1}$ plotted as a function of $T_{\dot{\gamma}}$. The inverse shear data have been multiplied by a constant factor 0.07 in order to align them with the $\tau_{\alpha}(T)$ data. The temperature dependence of the two quantities are in quite good agreement, suggesting a direct proportionality between them

$$
\tau_{\alpha}\left(T_{\dot{\gamma}}\right) \propto \dot{\gamma}^{-1}
$$

There is obviously a shear-independent prefactor that depends on the chosen definition of $\tau_{\alpha}$ and on the specific investigated correlation function (for example, the self relaxation time scale approximately as $q^{-2}$ ). It is interesting to note the existence of a simple linear relationship between $\tau_{\alpha}$ and $\dot{\gamma}^{-1}$ along the line $T_{\dot{\gamma}}$ in the $(T-\dot{\gamma})$ plane, as compared, for example, to the $\dot{\gamma}^{-2 / 3}$ dependence observed along the $T$-constant line [8].

Recent important work on the fluctuation-dissipation relation (FDR) in sheared system [3,86,10] has provided evidence that two different temperatures control the dynamics. Dynamics at short times is controlled by the bath temperature, while dynamics at longer times is controlled by an effective temperature, larger than the bath one. Our analysis predicts that only for $T<T_{\dot{\gamma}}$ a two-temperature scenario in the FDR should be observed.

Furthermore, our analysis confirms [7] that an interesting and promising (from a numerical and experimental point of view) implication of the above scenario could be the possibility to reproduce the aging properties of the non-driven system from the investigation of the shear 
effects on the driven system. The underling main hypothesis is the similarity between the properties of the non-driven system at a given waiting time and those of the driven system at a given shear rate $\dot{\gamma}$.

In conclusion we have numerically studied the shear flow effects on the thermal and dynamical properties of a simple model liquid, focusing on the differences between driven and equilibrium system, both in the dynamics and thermodynamics. The potential energy and the incoherent scattering function (and the associated structural relaxation time) have been studied as a function of temperature for different shear rates. It emerges the existence, for a given shear rate, of a crossover line $T_{\dot{\gamma}}$, separating two regimes: an high temperature (low shear rate) regime in which the driven system behaves very similar to the equilibrium one, and a low temperature (high shear rate) regime in which the driven system strongly deviate from the equilibrium one. From the temperature dependence of relaxation times we found further evidences that $T_{\dot{\gamma}}$ marks the temperature at which the shear relaxation times start to deviate from the equilibrium times, passing from an " $\alpha$-dominated" to a "shear-dominated" region and approaching a finite value at low temperature. Along the line defined by $T_{\dot{\gamma}}$ in the $(T-\dot{\gamma})$ plane we observe a direct proportionality between $\tau_{\alpha}$ and $\dot{\gamma}^{-1}$, allowing a more clear interpretation of the relationship between shear and relaxation times.

We acknowledge support from INFM Initiative Parallel Computing, and MURST COFIN2000. 


\section{REFERENCES}

[1] M. Utz, P.G. Debenedetti, and F.H. Stillinger, Phys. Rev. Lett. 84, 1471 (2000).

[2] R. Yamamoto and A. Onuki, Phys. Rev. E 58, 3515 (1998).

[3] L. Berthier and J.L. Barrat, J. Chem. Phys. 116, 6228 (2002).

[4] F.H. Stillinger and T.A. Weber, Phys. Rev. A 25, 978 (1982).

[5] D.L. Melandro and D.J. Lacks, Phys. Rev. Lett. 81, 5576 (1998).

[6] D.J. Lacks, Phys. Rev. Lett. 87, 225502 (2001).

[7] L.F. Cugliandolo, J. Kurchan and L. Peliti Phys. Rev. E 55, 3898 (1997).

[8] L. Berthier, J.L. Barrat, and J. Kurchan, Phys. Rev. E 61, 5464 (2000).

[9] J.P. Bouchaud, L.F. Cugliandolo, J. Kurchan and M. Mezard, Out of equilibrium dynamics in spin glasses and the other glassy systems, in Spin Glasses and Random Fields, edited by P. Young, World Scientific, Singapore, (1998).

[10] J.L. Barrat and L. Berthier, Phys. Rev. E 63, 012503 (2000).

[11] R. Di Leonardo , L. Angelani, G. Parisi and G. Ruocco, Phys. Rev. Lett. 84, 6054 $(2000)$.

[12] D.J. Evans and G.P. Morris, Statistical Mechanics of Nonequilibrium Liquids (Academic, London, 1990).

[13] B. Coluzzi, M. Mzard, G. Parisi, and P. Verrocchio J. Chem. Phys. 111, 9039 (1999).

[14] F. Sciortino, W. Kob and P. Tartaglia, Phys. Rev. Lett. 83, 3214 (1999).

[15] Y. Rosenfeld and P. Tarazona, Mol. Phys. 95, 141 (1998). Although derived for pair potentials of the type $\varphi(r) \propto r^{-n}$, the $T^{3 / 5}$ power law work very well also for the Lennard-Jones interaction [11, [13, 14]. 
[16] W. Götze, J. Phys.: Condens. Matter 11, A1 (1999).

[17] L. Angelani, R. Di Leonardo, G. Ruocco, A. Scala, and F. Sciortino, Phys. Rev. Lett. 85, 5356 (2000); J. Chem. Phys. (in press) (2002).

[18] C. De Michele and F. Sciortino, Phys. Rev. E (in press) (2002). 


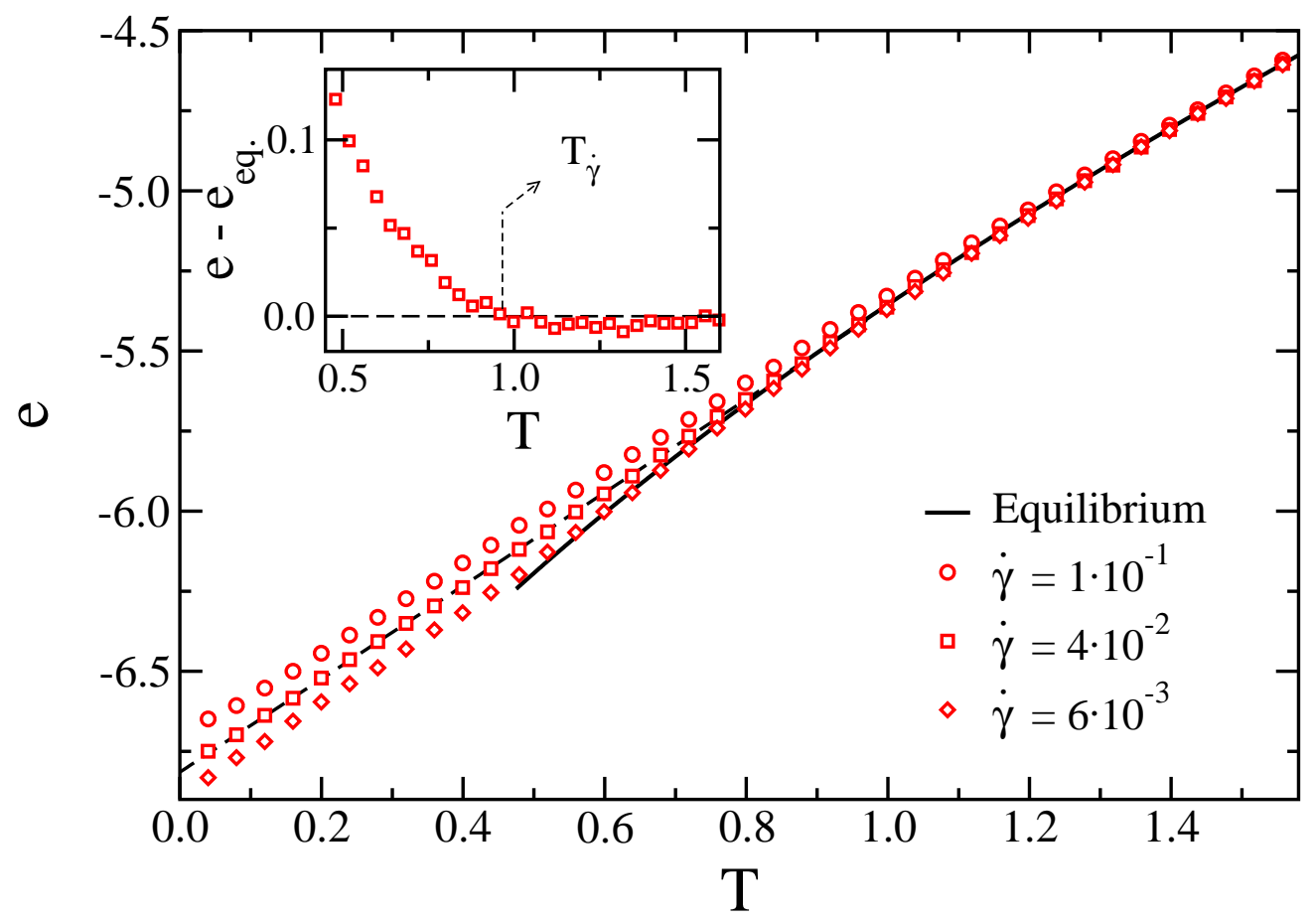

FIG. 1. Potential energy per particle $e$ as a function of temperature for the equilibrium system (full line) and for driven systems with different shear rates (symbols - from top to bottom decreasing $\left.\dot{\gamma}=1 \cdot 10^{-1}, 4 \cdot 10^{-2}, 6 \cdot 10^{-3}\right)$. The full line extends down to the lowest temperature that we are able to equilibrate in the simulation. The dashed line is the linear fit to the low temperature points of the $\dot{\gamma}=4 \cdot 10^{-2}$ curve. In the inset the difference between the energy curve for the shear $\dot{\gamma}=4 \cdot 10^{-2}$ and the $T^{3 / 5}$ fit of the equilibrium energy $e_{e q}$. as a function of $T$. The estimated crossover temperature $T_{\dot{\gamma}}$ is also indicated by the arrow. 


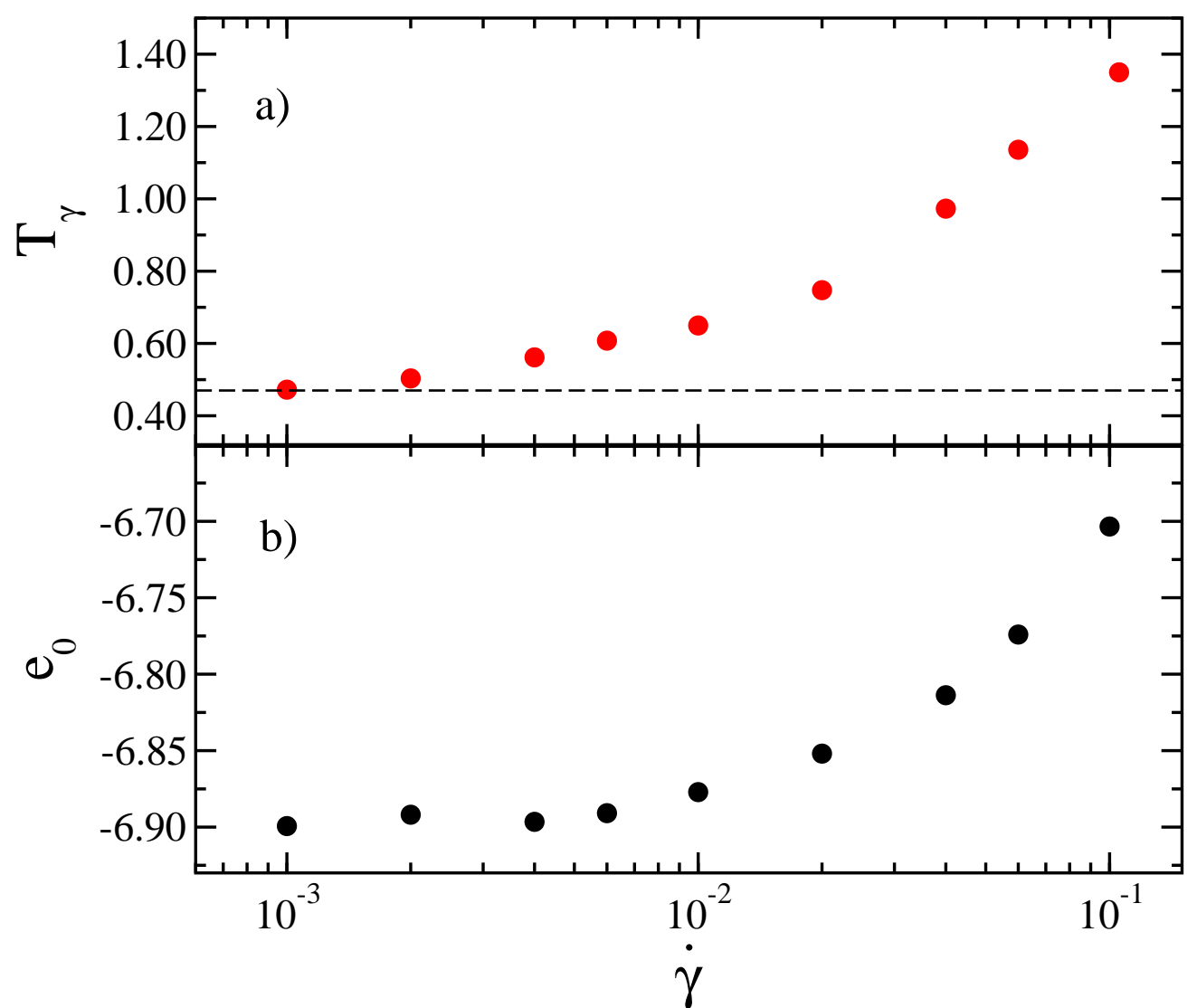

FIG. 2. The shear rate dependence of the parameters describing the energy curves of Fig. 1: a) crossover temperature $T_{\dot{\gamma}}$ (intersection between low energy linear behavior and $T^{3 / 5}$ high temperature dependence in Fig. 1 - the dashed line is the estimated value of the mode-coupling temperature $T_{M C T} \sim 0.475$ for this system), b) zero temperature energy value $e_{0}$ (intersection of energy curves with the $y$ axis in Fig. 1). 


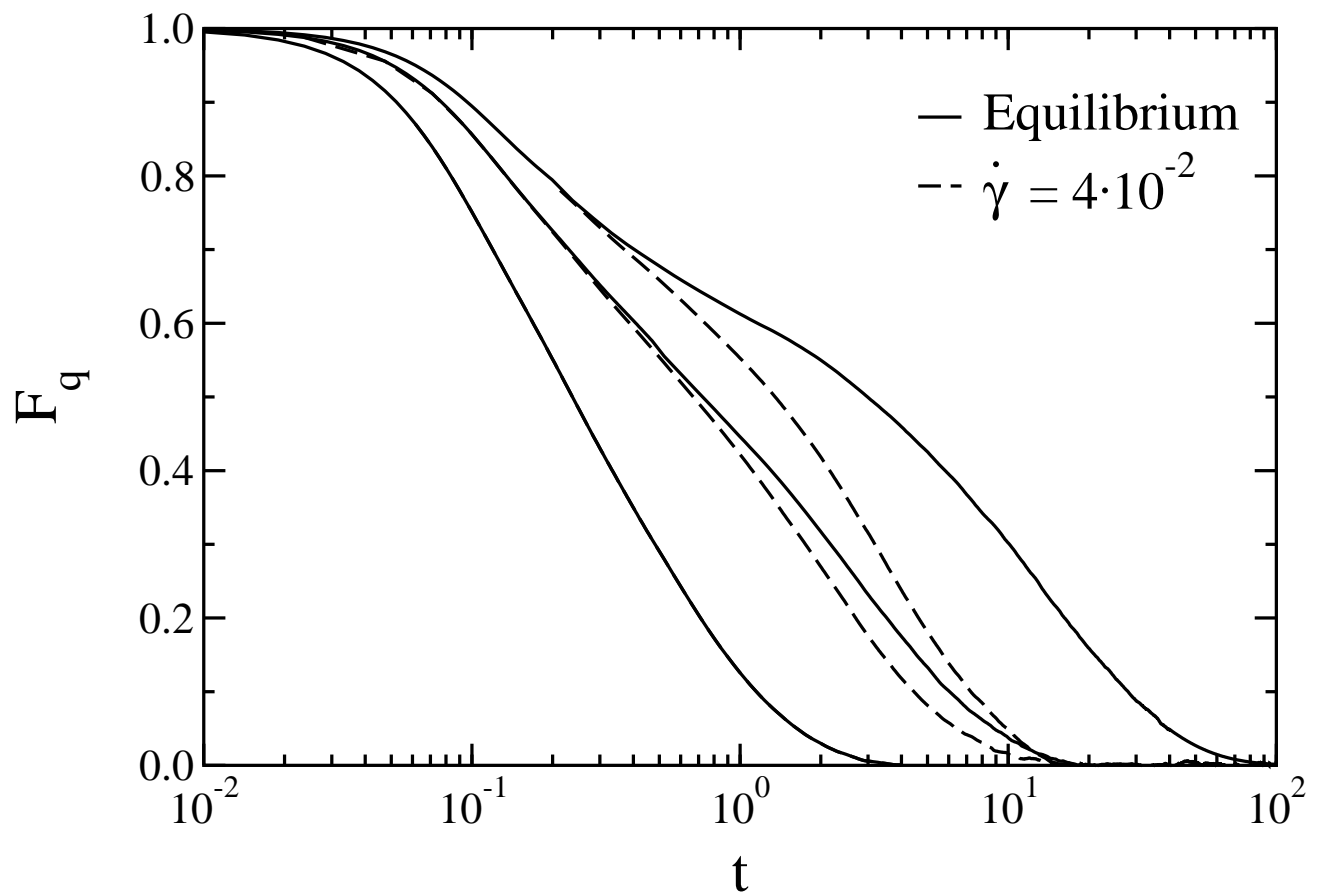

FIG. 3. Incoherent scattering functions $F_{\mathbf{q}}$ (calculated along the "shear-free" direction $z$ and for the $\mathbf{q}$ vector corresponding to the first peak of the static structure factor, $q_{\max }=7.1$ ) for the equilibrium system (full lines) and for the driven systems with shear rate $\dot{\gamma}=4 \cdot 10^{-2}$. The three curves refer to different temperatures: from left to right $T=1.6,0.80,0.56$ (we note that the crossover temperature $T_{\dot{\gamma}}$ as defined from Fig. 1 for the shear rate $\dot{\gamma}=4 \cdot 10^{-2}$ is $T_{\dot{\gamma}}=0.97$ ). 


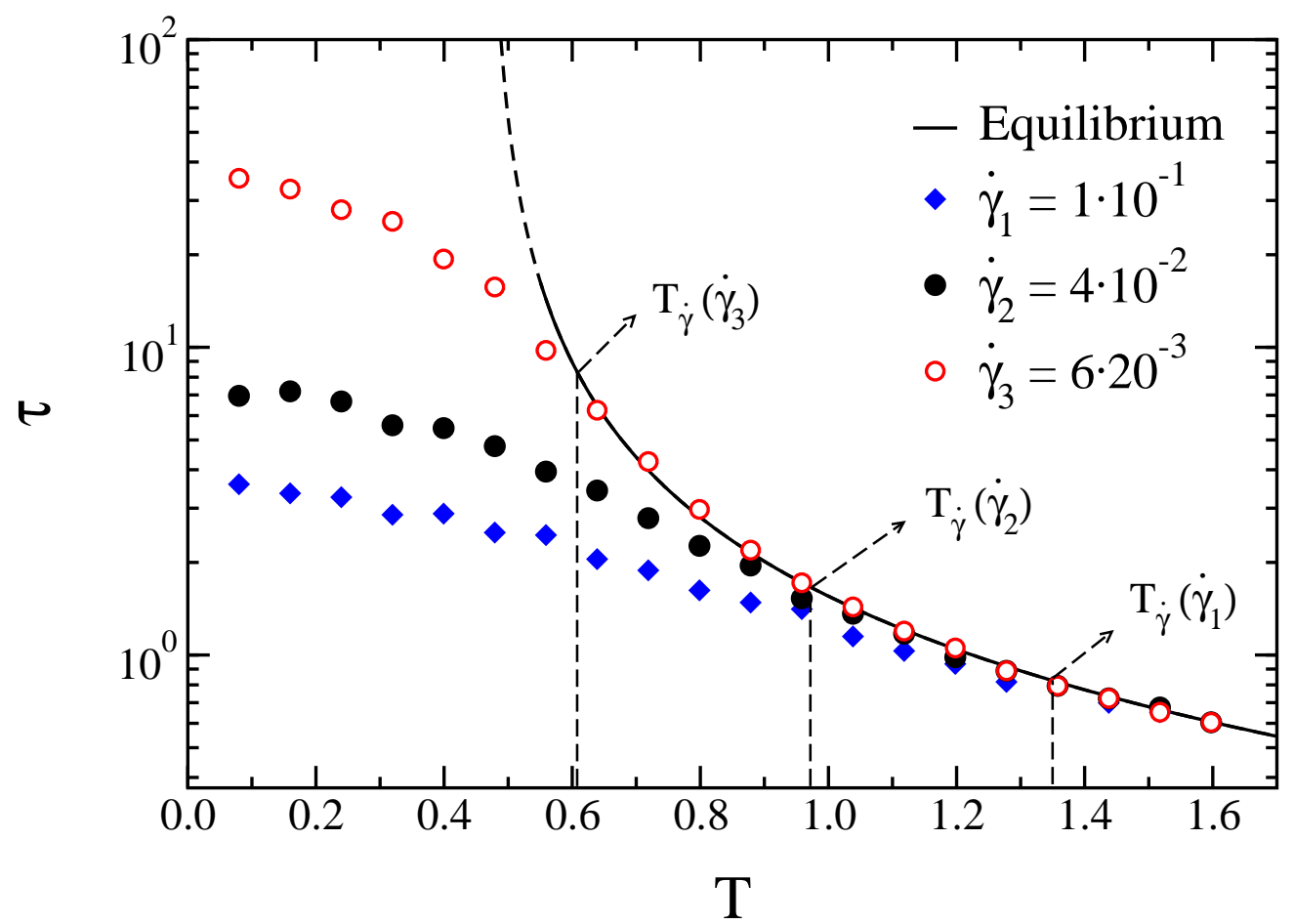

FIG. 4. Relaxation times (from incoherent scattering functions at $q_{\max }=7.1$, see Fig. 3 ) as a function of temperature for the equilibrium system (the full line is a power law fit of simulation data and dashed line is an extrapolation below the last simulation point) and for the driven systems with shear rates $\dot{\gamma}_{1}=1 \cdot 10^{-1}$ (full diamonds), $\dot{\gamma}_{2}=4 \cdot 10^{-2}$ (full circles) and $\dot{\gamma}_{3}=6 \cdot 10^{-3}$ (open circles). The arrows indicate the crossover temperatures $T_{\dot{\gamma}}$ as defined in Fig. 1 for the corresponding shear rates: $T_{\dot{\gamma}}\left(\dot{\gamma}_{1}\right)=1.35, T_{\dot{\gamma}}\left(\dot{\gamma}_{2}\right)=0.97$ and $T_{\dot{\gamma}}\left(\dot{\gamma}_{3}\right)=0.61$. 


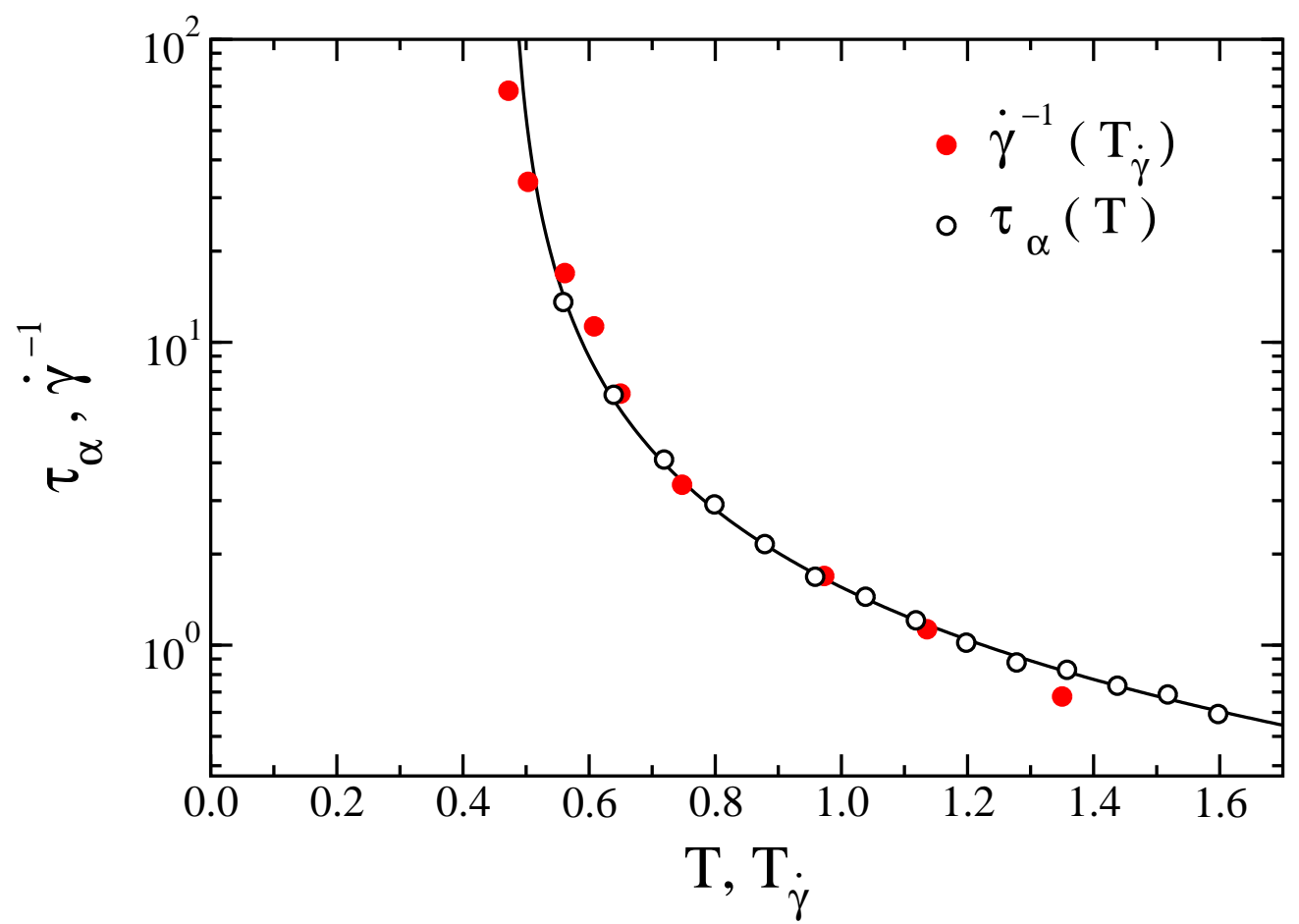

FIG. 5. Relaxation times $\tau_{\alpha}$ of the equilibrium system (open symbols) as a function of temperature (the full line is a power law fit), and the inverse shear rate $\dot{\gamma}^{-1}$ (full symbols - multiplied by an arbitrary factor 0.07 ) as a function of crossover temperature $T_{\dot{\gamma}}$ as defined from Fig. 1. 